\title{
Erratum to: Long-term outcome of epilepsy in patients with Prader-Willi syndrome
}

\author{
Alberto Verrotti - Raffaella Cusmai - Daniela Laino - Marco Carotenuto • Maria Esposito • \\ Raffaele Falsaperla $\cdot$ Lucia Margari $\cdot$ Renata Rizzo $\cdot$ Salvatore Savasta $\cdot$ Salvatore Grosso $\cdot$ \\ Pasquale Striano • Vincenzo Belcastro $\cdot$ Emilio Franzoni $\cdot$ Paolo Curatolo $\cdot$ Lucio Giordano • \\ Elena Freri $\cdot$ Sara Matricardi $\cdot$ Dario Pruna $\cdot$ Irene Toldo $\cdot$ Elisabetta Tozzi $\cdot$ Lucio Lobefalo • \\ Francesca Operto $\cdot$ Emma Altobelli $\cdot$ Francesco Chiarelli $\cdot$ Alberto Spalice
}

Published online: 27 November 2014

(c) Springer-Verlag Berlin Heidelberg 2014

\section{Erratum to: J Neurol \\ DOI 10.1007/s00415-014-7542-1}

Unfortunately, two co-author's names were not listed in the original publication. The missing author's names and affiliation are given below:

Marco Carotenuto

Maria Esposito

The online version of the original article can be found under doi:10.1007/s00415-014-7542-1.

\footnotetext{
A. Verrotti $(\bowtie) \cdot$ D. Laino

Department of Pediatrics, University of Perugia, Perugia, Italy

e-mail: averrott@unich.it

R. Cusmai

Neurology Unit, “'Bambino Gesù’’ Children's Hospital, IRCCS, Rome, Italy

M. Carotenuto $\cdot$ M. Esposito

Child and Adolescent Neuropsychiatry, Department of Mental

Health, Physical and Preventive Medicine, Second University

of Naples, Naples, Italy

R. Falsaperla

Pediatric Acute and Emergency Operative Unit and Department, Policlinico-Vittorio Emanuele University Hospital, University of Catania, Catania, Italy

L. Margari · F. Operto

Unit of Child Neuropsychiatry, Department of Basic Medical

Sciences, Neuroscience and Sense Organs, "Aldo Moro"

University of Bari, Bari, Italy

R. Rizzo

Section of Child Neuropsychiatry, Department of Pediatrics,

University of Catania, Catania, Italy
}

Child and Adolescent Neuropsychiatry, Department of Mental Health, Physical and Preventive Medicine, Second University of Naples, Naples, Italy. 
L. Giordano

Pediatric Neuropsychiatric Division, “'Ospedali Civili”, Brescia, Italy

E. Freri

Department of Pediatric Neuroscience, Foundation I.R.C.C.S. Neurological Institute C. Besta, Milan, Italy

S. Matricardi $\cdot$ F. Chiarelli

Department of Pediatrics, University of Chieti, Chieti, Italy

D. Pruna

Division of Child Neurology, Department of Pediatrics,

University "La Sapienza" Rome, Rome, Italy

I. Toldo

Pediatric Neurology Unit, Department of Women's and

Children's Health, University of Padova, Padua, Italy

E. Tozzi

Department of Pediatrics, University of L'Aquila, L'Aquila,

Italy
L. Lobefalo

Section of Ophthalmology, Department of Experimental and Clinical Sciences, University of Chieti, Chieti, Italy

E. Altobelli

Department of Life, Health and Environmental Sciences, University of L'Aquila, L'Aquila, Italy

\section{A. Spalice}

Chair of Pediatrics, Child Neurology, II Faculty of Medicine, University of Rome, Rome, Italy 